\title{
Epigenetic regulation of miRNA-124 and multiple downstream targets is associated with treatment response in myeloid malignancies
}

\author{
HONGBIN LIU ${ }^{1,2}$, PHILLIP PATTIE ${ }^{1,2}$, SAHAN CHANDRASEKARA ${ }^{3}$, \\ ANDREW SPENCER ${ }^{1}$ and ANTHONY E. DEAR ${ }^{1-3}$
}

${ }^{1}$ Australian Centre for Blood Diseases, Monash University, Melbourne, Victoria 3004; ${ }^{2}$ Translational Research Division,
Eastern Clinical Research Unit, Australian Centre for Blood Diseases, Monash University, Melbourne, Victoria 3004;
${ }^{3}$ Department of Medicine, Eastern Health Clinical School, Monash University, Melbourne, Victoria 3128, Australia

Received April 28, 2016; Accepted July 7, 2016

DOI: $10.3892 / \mathrm{ol} .2016 .4912$

\begin{abstract}
Epigenetic regulation of microRNA (miRNA) expression has recently been implicated in the pathogenesis of myelodysplastic syndrome (MDS). Particular interest has focused on miRNA-124 expression, which is inhibited in MDS and has recently been demonstrated to be upregulated in response to epigenetic treatment (EGT). Previous studies have determined the in vitro and in vivo expression of miRNA-124 and several molecular targets, including cyclin-dependent kinase (CDK) 4, CDK6 and enhancer of zeste homolog 2 (EZH2), in order to elucidate the molecular mechanisms associated with the miRNA-124-mediated therapeutic response to EGT in MDS and identify additional potential biomarkers of early EGT treatment response in myeloid malignancies. In vitro studies in the HL60 leukemic cell line identified upregulation of miRNA-124 expression in response to single-agent EGT with either azacytidine (AZA) or the histone deacetylase inhibitor panobinostat (LBH589). Combination EGT with AZA and LBH589 resulted in significant additive induction of miRNA-124 expression. Expression of downstream targets of miRNA-124, including CDK4, CDK6 and EZH2, in response to single agent and combined EGT was determined in HL60 cells. Single and combination EGT treatment resulted in inhibition of CDK4, CDK6 and EZH2 expression with combination EGT resulting in a significant and additive inhibitory effect. In vivo studies using peripheral blood mononuclear cells from patients receiving combination EGT for high risk MDS or acute myeloid leukemia demonstrated significant induction of miRNA-124 and inhibition CDK4 and CDK6 messenger (m)RNA expression
\end{abstract}

Correspondence to: Dr Anthony E. Dear, Department of Medicine, Eastern Health Clinical School, Monash University, Level 2, 5 Arnold Street, Melbourne, Victoria 3128, Australia E-mail: anthony.dear@monash.edu.au

Key words: epigenetic, miRNA, myelodysplasia, myeloid leukemia, biomarker in patients that responded to combination EGT. A trend to inhibited EZH2 mRNA expression was also identified in response to combination EGT. Overall, the present observations identify a potential molecular mechanism for miRNA-124-mediated response to EGT involving regulation of CDK4, CDK6 and EZH2 expression. In addition, the present findings further qualify miRNA-124 as a possible biomarker of early response to EGT in myeloid malignancies and potentially a valid therapeutic target, together with CDK4, CDK6 and EZH2.

\section{Introduction}

Epigenetic mechanisms, including microRNAs (miRNAs), have been implicated in the pathogenesis and prognosis of myelodysplastic syndrome (MDS) and have been exploited in the successful treatment of this condition (1-2). Single-agent epigenetic therapy (EGT) with demethylating agents, including azacytidine (AZA) and decitabine, has been successfully utilized in MDS to improve overall survival rate and time (1-3), whilst clinical trials suggest combination EGT utilizing demethylating agents and histone deacetylase inhibitors (HDACis) may result in enhanced clinical responses over single-agent therapy (4). Despite successful therapeutic intervention utilizing EGT in MDS, not all patients respond $(\sim 50 \%)$, and treatment regimens are often lengthy, with responses frequently only observed subsequent to several cycles of therapy (3). The problematic response to EGT in MDS has generated a significant demand for molecular markers to improve the early prediction of response to EGT.

miRNAs are small non-coding single stranded RNA molecules of 20-25 base pairs that are responsible for post-transcriptional regulation of gene expression (5). Epigenetic regulation of miRNA expression has recently been implicated in the pathogenesis of MDS. Demonstration of epigenetic silencing of the miRNA-124 promoter in murine MDS studies (6) together with significant inhibition of miRNA-124 expression and excessive miRNA-124 promoter methylation in MDS patients (7) suggests miRNA-124 may not only be implicated in the pathogenesis of MDS, but also potentially in the molecular mechanisms 
associated with response to EGT. Evidence of early upregulation of miRNA-124 expression in patients with MDS and acute myeloid leukemia (AML) treated with and responding to single-agent or combination EGT compared with non-responding patients suggests a critical role for epigenetic regulation of miRNA-124 in the response to EGT and indicates a potential use of miRNA-124 as a biomarker of early response to EGT in MDS (8).

Investigation into downstream molecular targets of EGT-mediated 're-expression' of miRNA-124 may reveal additional molecular mechanisms associated with the EGT-mediated miRNA-124 therapeutic response in MDS and afford the potential for identification of additional biomarkers and therapeutic targets for this condition. Previous studies have demonstrated that epigenetic loss of miRNA-124 expression correlates with activation of the oncogene cyclin-dependent kinase (CDK) 6, which in turn phosphorylates and inactivates the tumor-suppressor protein $\mathrm{Rb}$ (9). EGT-mediated induction of miRNA-124 in MDS/AML is associated with attenuation of CDK6 messenger (m) RNA levels in treatment responders (8), suggesting a tumor suppressor role for miRNA-124.

The present study aimed to evaluate the effects of combination EGT with AZA and panobinostat (LBH589) in vitro and in vivo on expression of additional downstream targets of miRNA-124, including CDK4 and enhancer of zeste homolog 2 (EZH2) in order to further elucidate the molecular mechanisms associated with EGT-mediated regulation of miRNA-124 expression in MDS/AML. The present study aimed to further qualify miRNA-124 as a possible biomarker of early response to EGT and potentially a valid therapeutic target, together with CDK4, CDK6 and EZH2.

\section{Materials and methods}

Cell culture. HL60 cells were cultured in RPMI-1640 (Gibco; Thermo Fisher Scientific, Inc., Waltham, MA, USA) containing $10 \%$ heat-inactivated fetal calf serum (Thermo Fisher Scientific, Inc.) and kept in a $5 \% \mathrm{CO}_{2}$ incubator at $37^{\circ} \mathrm{C}$. The agents added to plates for $48 \mathrm{~h}$ were as follows: AZA, which was provided by Celgene (Melbourne, Australia); and LBH589, which was provided by Novartis (Sydney, Australia). AZA was dissolved in $\mathrm{H}_{2} \mathrm{O}$ with $0.2 \%$ acetic acid and used at a final concentration of $1.0 \mu \mathrm{M}$. LBH589 was dissolved in phosphate-buffered saline (PBS) with $1 \%$ dimethyl sulfoxide and used at a final concentration of $20 \mathrm{nM}$.

RNA extraction. TRIzol (Invitrogen; Thermo Fisher Scientific, Inc.) was used to extract RNA from HL60 cells treated with AZA, LBH589 or a combination of AZA and LBH589. TRIzol was also used to extract RNA from the mononuclear cell fractions of blood samples obtained from 9 patients in a phase Ib/II clinical trial (10) and healthy controls $(n=6)$. The open-label, phase Ib/II study (10) was conducted at three centres: Alfred Hospital, Melbourne, Australia; Princess Alexandra Hospital, Brisbane, Australia; and Austin Hospital, Melbourne, Australia. The study was approved by the ethics committees at the aforementioned institutions and was performed in accordance with the principles of independent Human Research and Ethics Committees, and registered with the Australian and New Zealand Clinical Trials Registry (ACTRN12610000924055). All participating patients and healthy controls were required to provide written informed consent for participation in the study.

The clinical trial consisted of a 5-day schedule of AZA administration followed by LBH589 administration in high-risk MDS or AML patients. On days 1-5, $75 \mathrm{mg} / \mathrm{m}^{2}$ AZA was injected subcutaneously and LBH589 was administered orally 3 times a week (Monday/Wednesday/Friday), starting on day 5 of each 28-day cycle. A total of 40 evaluable patients were enrolled in the present study (10), of which, 9 patients, consisting of 5 patients with AML and 4 patients with high-risk MDS (Table I), were evaluated for miRNA-124, CDK4, CDK6 and EZH2 expression at screen and prior to the end of cycle 1 (day 25). The association between the expression of miRNA-124, CDK4, CDK6 and EZH2 and treatment response were determined using bone marrow biopsies at 1 , 3 and 6 months subsequent to treatment commencement (10). Bone marrow biopsies were performed at 1, 3 and 6 months after treatment initiation to evaluate the clinical response to treatment, which may be delayed with epigenetic treatment. Only end of first cycle (day 25) miRNA-124, CDK4, CDK6 and EZH2 samples were evaluated, as the study was investigating early markers of response to epigenetic treatment. Treatment responses were defined according to International Working Group criteria for AML and MDS (11).

Reverse transcription (RT). RT of mRNA obtained from HL60 cells, healthy volunteers and patients was performed using a $15 \mu \mathrm{l}$ reaction mix containing $100 \mathrm{mM}$ deoxynucleotide triphosphates (dNTPs), $50 \mathrm{U} / \mu 1$ MultiScribe Reverse Transcriptase, 10X RT buffer, $20 \mathrm{U} / \mu 1$ RNase Inhibitor, nuclease-free water (Invitrogen; Thermo Fisher Scientific, Inc.), oligo(dT) random primer (Promega Corporation, Madison, WI, USA) and mRNA samples from HL60 cells, healthy volunteers or patients.

RNA isolation and stem-loop RT-polymerase chain reaction (PCR) for miRNA-124. Total RNA was isolated using TRIzol, according to the manufacturer's protocol. RT was performed using TaqMan MicroRNA RT kit and TaqMan MicroRNA Assay kit (Applied Biosystems; Thermo Fisher Scientific, Inc.), according to the manufacturer's protocol. Total RNA was reverse transcribed using $1 \mathrm{mM}$ dNTPs, $50 \mathrm{U}$ MultiScribe Reverse Transcriptase, 1X RT Buffer, 3.8 U RNase Inhibitor and 1X stem-loop RT primer (all obtained from Applied Biosystems; Thermo Fisher Scientific, Inc.), under the following thermal cycling conditions: $16^{\circ} \mathrm{C}$ for $30 \mathrm{~min} ; 42^{\circ} \mathrm{C}$ for $30 \mathrm{~min}$; and $85^{\circ} \mathrm{C}$ for $5 \mathrm{~min}$. Quantitative PCR (qPCR) of miRNA-124 was performed using $1.33 \mathrm{ml}$ of 1:15 diluted RT product in 1X TaqMan Universal PCR Master Mix (Applied Biosystems; Thermo Fisher Scientific, Inc.) and 1X TaqMan Assay (Applied Biosystems; Thermo Fisher Scientific, Inc.) at $95^{\circ} \mathrm{C}$ for $10 \mathrm{~min}$, followed by 40 cycles of $95^{\circ} \mathrm{C}$ for $15 \mathrm{sec}$ and $60^{\circ} \mathrm{C}$ for $1 \mathrm{~min}$. U6 was used as a reference for data analysis using the $2^{-\Delta \Delta \mathrm{Cq}}$ method (12).

$q P C R$. Reaction volumes of $20 \mu \mathrm{l}$ contained SYBR Green 1 Buffer (Qiagen GmbH, Hilden, Germany) and forward and reverse primers for the target genes CDK4, CDK6 and EZH2. Each PCR run also included wells containing a no-template 
Table I. Association between clinical and molecular response and miRNA-124, CDK4, CDK6 and EZH2 expression.

\begin{tabular}{ccccccccc}
\hline $\begin{array}{l}\text { Patient } \\
\text { ID }\end{array}$ & $\begin{array}{c}\text { Age, } \\
\text { years }\end{array}$ & Gender & Diagnosis & $\begin{array}{c}\text { Best } \\
\text { response }\end{array}$ & $\begin{array}{c}\text { miRNA-124 } \\
\text { induction }^{\mathrm{a}}\end{array}$ & $\begin{array}{c}\text { CDK4 } \\
\text { inhibition }^{\mathrm{b}}\end{array}$ & $\begin{array}{c}\text { CDK6 } \\
\text { inhibition }^{\mathrm{b}}\end{array}$ & $\begin{array}{c}\text { EZH2 } \\
\text { inhibition }^{\mathrm{c}}\end{array}$ \\
\hline 3 & 72 & F & MDS & CR & Yes & Yes & Yes & Yes \\
7 & 58 & M & AML & Resistant & No & No & No & No \\
8 & 75 & M & AML & Resistant & No & No & No & No \\
18 & 67 & F & MDS & PR & No & Yes & Yes & Yes \\
22 & 78 & M & AML & CR & Yes & No & No & No \\
23 & 69 & F & AML & Resistant & No & No & No & Yes \\
26 & 75 & M & MDS & PD & No & No & No & Yes \\
28 & 69 & M & AML & PR & Yes & Yes & Yes & Yes \\
33 & 62 & F & MDS & Marrow CR & Yes & Yes & Yes & Yes \\
\hline
\end{tabular}

${ }^{\mathrm{a}}$ miRNA-124 2.0-fold or greater induction over screen. ${ }^{\mathrm{b}} \mathrm{CDK} 4, \mathrm{CDK} 6$ 1.0-fold or greater inhibition over screen. ${ }^{\mathrm{c}} \mathrm{EZH} 2$ 2.0-fold or greater inhibition over screen. miRNA, microRNA; CDK, cyclin-dependent kinase; EZH2, enhancer of zeste homolog 2.

control. A melting point dissociation curve generated by the instrument (Applied Biosystems 7500 Real-Time PCR System; Thermo Fisher Scientific, Inc.) was used to confirm that only a single product was present. The fluorescence data were quantitated using the threshold cycle value (12). Data were normalized to $\beta$-actin and presented as the mean fold-change compared with the pre-treatment screening sample. PCR was performed a minimum of 2 times for each patient sample to ensure consistency (13). Forward and reverse primer sequences for CDK4, CDK6 and EZH2 were as follows: CDK4 forward, 5'-AGTTCGTGAGGTGGCTTTA-3' and reverse, 5'-GGG TGCCTTGTCCAGATA-3'; CDK6 forward, 5'-GCCTAT GGGAAGGTGTTCAA-3' and reverse, 5'-CTGTCTGTTCGT GACACTGT-3'; and EZH2 forward, 5'-TTCATGCAACAC CCAACACT-3' and reverse, 5'-GAGAGCAAACTCCT-3'. The primers used for $\beta$-actin were as follows: Forward, 5'-GAC AGGATGCAGAAGGAGATTACT-3'; and reverse, 5'-TGA TCCACATCTGCTGGAAGGT-3'.

Statistical analysis. Results were expressed as the mean + standard error of the mean, and analyzed using GraphPad Prism 5 software (GraphPad Software, Inc., La Jolla, CA, USA), using $t$-tests for two-group comparisons and one-way analysis of variance for three or more group comparisons. $\mathrm{P}<0.05$ was considered to indicate a statistically significant difference.

\section{Results}

Effect of AZA and LBH589 on miRNA-124, CDK4, CDK6 and EZH2 expression in HL60 cells. LBH589 or AZA alone increased miRNA-124 expression, with AZA being the more potent agent (Fig. 1A). The combination of AZA with LBH589 demonstrated a significant and additive increase in miRNA-124 expression compared with single-agent treatment (Fig. 1A). LBH589 or AZA alone decreased expression of CDK4 expression and the combination of AZA and LBH589 significantly and additively decreased CDK4 expression compared with single agent treatment (Fig. 1B). AZA and LBH589 inhibited CDK6 expression, with LBH589 being the more potent agent and demonstrating a significant inhibition of CDK6 expression (Fig. 1C). The combination of AZA and LBH589 also resulted in significant attenuation of CDK6 mRNA expression compared with untreated cells (Fig. 1C). AZA, LBH589 and the combination of AZA and LBH589 all significantly inhibited EZH2 expression, with single and combination therapy being equally efficacious (Fig. 1D).

In vivo expression of miRNA-124, CDK4, CDK6 and EZH2 in peripheral blood mononuclear cells. miRNA-124, CDK4, CDK6 and EZH2 mRNA expression levels were determined at screening (prior to treatment commencement, day 0) and on day 25, subsequent to the first cycle of treatment. Screening levels of miRNA-124, CDK4, CDK6 and EZH2 expression were initially compared between patients and healthy controls (Fig. 2A-D). A decrease in miRNA-124 and increase in CDK4, CDK6 and EZH2 expression was observed in patients compared with controls, suggesting disease-associated inhibition of miRNA-124 and induction of CDK4, CDK6 and EZH2.

Subsequent evaluation of miRNA-124, CDK4, CDK6 and EZH2 expression levels was performed to compare treatment non-responders and responders at screening (Fig. 3A-D). No significant differences were observed between responder and non-responder expression levels of miRNA-124, CDK4, CDK6 and EZH2 at screening (Fig. 3).

Finally, post-first treatment cycle evaluation of miRNA-124, CDK4, CDK6 and EZH2 mRNA expression levels between treatment responders and non-responders was performed (Fig. 4A-D). A significant increase in the expression of miRNA-124 compared with screening levels (Figs. 3A and 4A), together with a significant association with treatment response, compared with non-responders at day 25, was observed in the responders (Fig. 4A). CDK4, CDK6 and EZH2 mRNA expression in responders at day 25 subsequent to the first cycle of treatment was decreased compared with screening levels in responders (Figs. 3B-D and 4B-D). Notably, a significant decrease in CDK4 and CDK6 was observed, although significant inhibition of EZH2 expression was not observed, in treatment responders compared to non-responders at day 25 (Fig. 4B-D; Table I). 

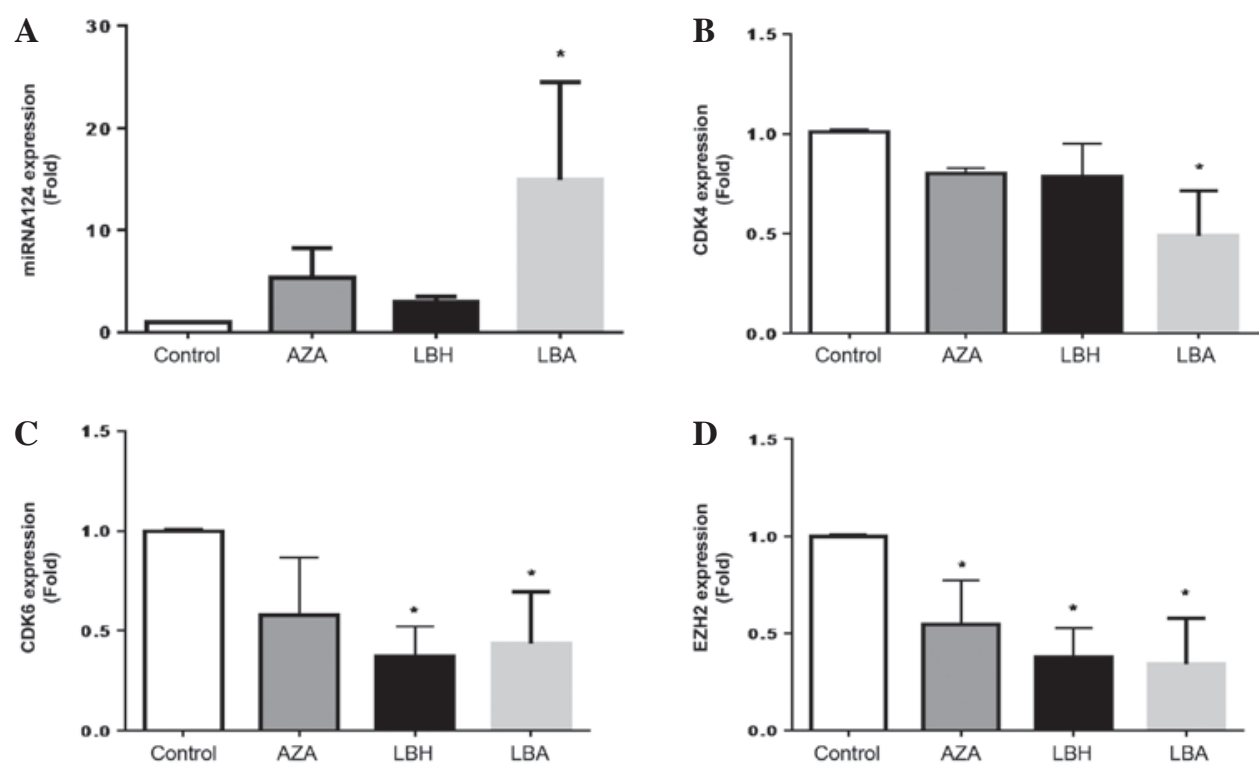

Figure 1. In vitro effects of azacytidine and/or LBH589 on (A) miRNA-124, (B) CDK4, (C) CDK6 and (D) EZH2 mRNA expression in HL60 cells. ${ }^{*} \mathrm{P}<0.05$ vs. Control $(\mathrm{n}=3)$. The P-values obtained were as follows: (A) $\mathrm{P}=0.620,0.740$ and 0.032 for AZA, $\mathrm{LBH}$ and LBA, respectively, vs. Control; (B) $\mathrm{P}=0.812$, 0.511 and 0.025 for AZA, LBH and LBA, respectively, vs. Control; (C) P=0.519, 0.024 and 0.031 for AZA, LBH and LBA, respectively, vs. Control; and (D) $\mathrm{P}=0.025,0.002$ and 0.008 for AZA, LBH and LBA, respectively, vs. Control. LBH589, panobinostat; miRNA, microRNA; CDK, cyclin-dependent kinase; EZH2, enhancer of zeste homolog 2; AZA, cells treated with $1.0 \mu \mathrm{M} 5$-azacytidine for 48 h; LBH, cells treated with $20 \mathrm{nM} \mathrm{LBH} 589$ for 48 h; LBA, cells treated with $20 \mathrm{nM}$ LBH589 + $1.0 \mu \mathrm{M}$ AZA; Control, HL60 cells without treatment.
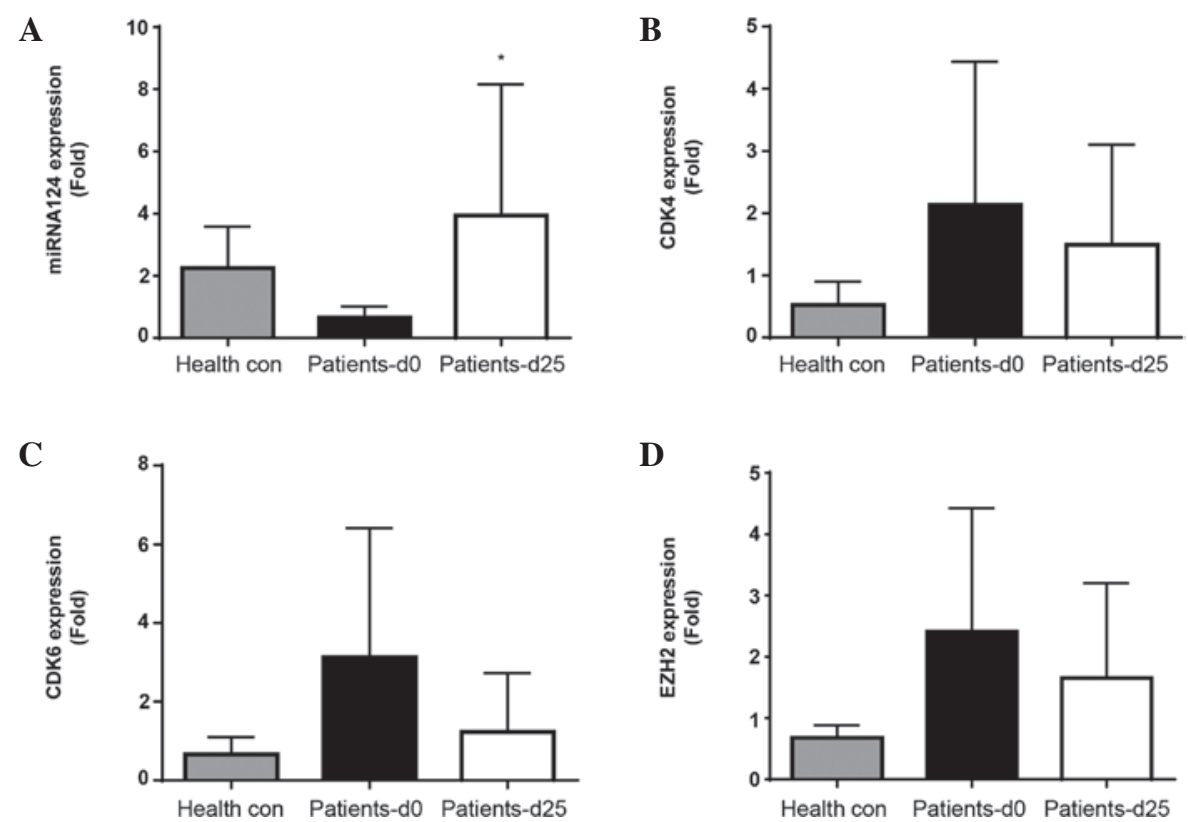

Figure 2. In vivo expression of miRNA-124, CDK4, CDK6 and EZH2 in healthy controls and patients with myelodysplastic syndrome or acute myeloid leukemia. Expression of (A) miRNA-124, (B) CDK4, (C) CDK6 and (D) EZH2 in healthy controls (Health con) and from patients (combined responders and non-responders) at screening (Patients-d0) and at day 25 (Patients-d25) when treated with a combination of azacytidine and panobinostat. "P<0.032 for patients at day 25 vs. patients at screening ( $\mathrm{n}=6$ healthy controls and 9 patients); $\mathrm{P}=0.055$ for patients at day 0 vs. healthy controls ( $\mathrm{n}=6$ healthy controls and 9 patients). miRNA, microRNA; CDK, cyclin-dependent kinase; EZH2, enhancer of zeste homolog 2.

\section{Discussion}

Epigenetic dysregulation of miRNAs is an emerging mechanism of action implicated in the pathogenesis, response to therapy and prognosis of myeloid malignancies (1). miRNA-124, a potential tumor suppressor gene with a hypothesized epigenetic role in the development of MDS and AML, has previously been shown to respond to EGT and may have a role in the therapeutic response to EGT in MDS/AML, with potential to act as a biomarker of early treatment response $(1,6-8)$.

Downstream targets of EGT-mediated miRNA-124 re-expression identified using either single agent or combined EGT include repression of CDK4, CDK6 and EZH2 mRNA, resulting in in vitro and in vivo inhibition of cell growth in hematological malignancies and solid tumors $(8,9,14-16)$. The present study has confirmed and extended these observations 

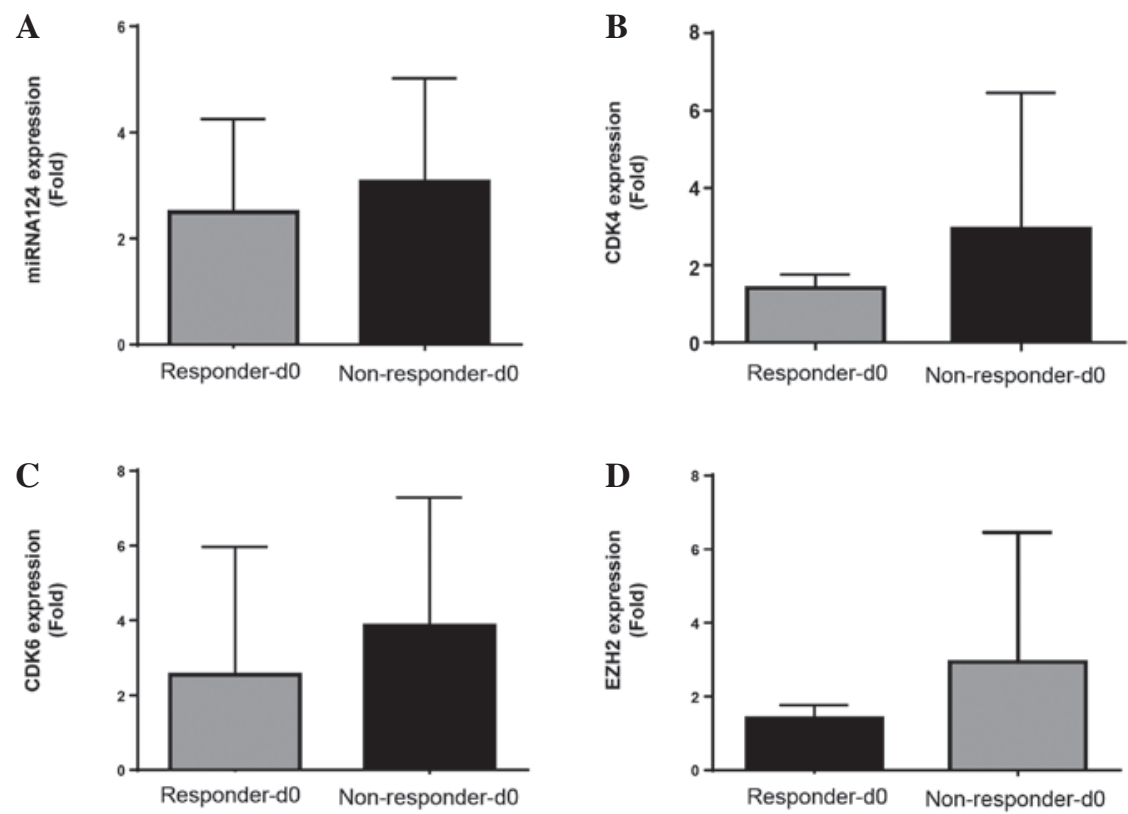

Figure 3. In vivo expression at screening of miRNA-124, CDK4, CDK6 and EZH2 from responder and non-responder MDS/AML patients treated with a combination azacytidine and panobinostat. Pre-treatment screening mRNA expression levels of (A) miRNA-124, (B) CDK4, (C) CDK6 and (D) EZH2 for responders (Responders-d0) and non-responders (Non-responder-d0), with clinical response to treatment determined at 1,3 and 6 months $(\mathrm{n}=9)$. Responses were defined according to International Working Group criteria for AML and MDS (11). AML, acute myeloid leukemia; MDS, myelodysplastic syndrome; miRNA, microRNA; CDK, cyclin-dependent kinase; EZH2, enhancer of zeste homolog 2.
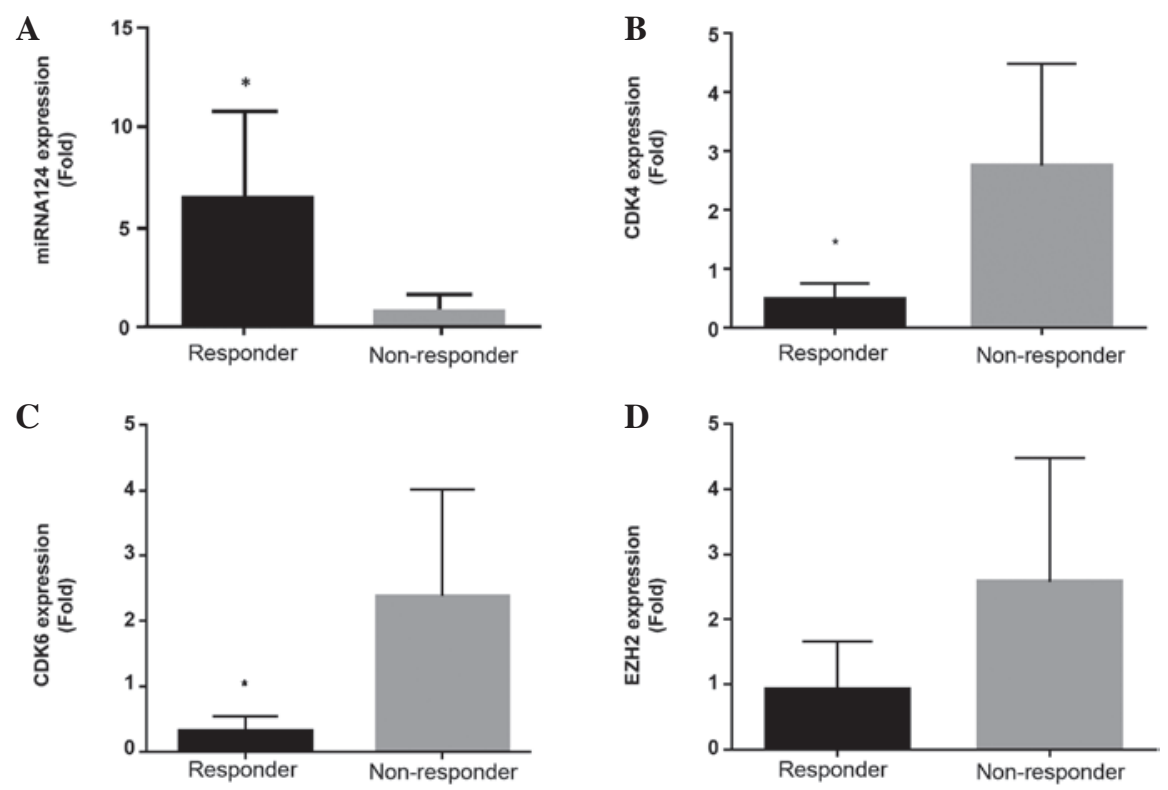

Figure 4. In vivo expression at Day 25 of miRNA-124, CDK4, CDK 6 and EZH2 from responder and non-responder MDS/AML patients treated with a combination of azacytidine and panobinostat. mRNA expression levels of (A) miRNA-124, (B) CDK4, (C) CDK6 and (D) EZH2 subsequent to first cycle (day 25) for responders and non-responders, with clinical response to treatment determined at 1,3 and 6 months $(n=9)$. $P<0.05$, responder vs. non-responder. The P-values were as follows: (A), $\mathrm{P}=0.036$; (B) $\mathrm{P}=0.036$; (C) $\mathrm{P}=0.025$; and (D) $\mathrm{P}=0.111$. Responses were defined according to International Working Group criteria for AML and MDS (11). MDS, myelodysplastic syndrome; AML, acute myeloid leukemia; miRNA, microRNA; CDK, cyclin-dependent kinase; EZH2, enhancer of zeste homolog 2 .

to delineate multiple downstream targets of increased miRNA-124 expression in MDS/AML and associate this with response to combination EGT.

In vitro studies in the HL60 cell line confirm that EGT-mediated re-expression of miRNA-124 with either single-agent or combination EGT is associated with repression of multiple targets critical to cell cycle progression, such as CDK4 and CDK6, and the oncoprotein EZH2. Notably, EZH2, a histone methyl transferase, has been identified to mediate oncogenic and tumor suppressor effects in myeloid malignancies $(1,17)$. Inactivating mutations of EZH2 have been identified in MDS (1), whilst loss of function mutations in EZH2 are also associated with a decreased rate of progression to AML (18). EZH2 upregulation has also been identified 
in myeloproliferative disease (19) and AML (20). EZH2 (21), CDK4 and CDK6 (22) have all been identified as potential therapeutic targets in hematological malignancies and may therefore have a potential mechanistic role in EGT treatment response. Additional studies are required to fully elucidate the roles of CDK4, CDK6 and EZH2 in this setting.

In vivo analysis of miRNA-124 expression together with its downstream targets CDK4, CDK6 and EZH2 in patients identified significantly reduced expression of miRNA-124 in patients compared with healthy controls (Fig. 2), in addition to upregulation of CDK4, CDK6 and EZH2, suggesting these expression changes are integral to disease pathogenesis. Screening levels of miRNA-124, CDK4, CDK6 or EZH2 demonstrated no significant association with clinical response to therapy (Fig. 3), similar to several previous studies undertaking analysis of other epigenetic markers in combination EGT $(10,23)$. Determination of miRNA-124, CDK4 and CDK6 expression levels from the post-first cycle treatment time point (day 25), demonstrated a significant association with clinical response, although EZH2 showed a trend to but no significant association (Fig. 4; Table I). CDK4, CDK6 and EZH2 have been previously identified as potential targets of upregulation of miRNA-124 expression in uveal melanoma (16). This suggests a canonical pathway in response to miRNA-124 upregulation in the setting of oncogenic transformation. The lack of association between clinical response to EGT and miRNA-124, CDK4, CDK6 and EZH2 expression levels at screening suggests that these molecules would not be useful predictors of clinical response if used prior to commencement of EGT. However, they may be useful as early as subsequent to the first cycle of treatment, resulting in significant improvement over current predictive strategies for determining early response to EGT.

Overall, the present observations provide a potential molecular mechanism for miRNA-124-mediated response to EGT in patients receiving combination treatment with a demethylating agent and HDACi for high-risk MDS/AML, further qualifying miRNA-124 as a possible marker of early response to EGT and potentially a valid therapeutic target, together with CDK4, CDK6 and EZH2. Future studies may include evaluation of miRNA-124 in comparison to other potential markers of early treatment response, such as orphan nuclear receptor NUR77, and confirmation of EZH2 expression and subsequent prognosis in response to EGT.

\section{References}

1. Vasilatou D, Papageorgiou SG, Dimitriadis G and Pappa V: Epigenetic alterations and microRNAs: New players in the pathogenesis of myelodysplastic syndromes. Epigenetics 8: 561-570, 2013

2. Tan PT and Wei AH: The epigenomics revolution in myelodysplasia: A clinico-pathological perspective. Pathology 43: 536-546, 2011.

3. Fenaux P, Mufti GJ, Hellstrom-Lindberg E, Santini V, Finelli C, Giagounidis A, Schoch R, Gattermann N, Sanz G, List A, et al: Efficacy of azacitidine compared with that of conventional care regimens in the treatment of higher-risk myelodysplastic syndromes: A randomised, open-label, phase III study. Lancet Oncol 10: 223-232, 2009 .

4. Ornstein MC, Mukherjee S and Sekeres MA: More is better: Combination therapies for myelodysplastic syndromes. Best Pract Res Clin Haematol 28: 22-31, 2015.

5. Chen CZ: MicroRNAs as oncogenes and tumor suppressors. N Engl J Med 353: 1768-1771, 2005.
6. Dickstein J, Senyuk V, Premanand K, Laricchia-Robbio L, Xu P, Cattaneo F, Fazzina R and Nucifora G: Methylation and silencing of miRNA-124 by EVI1 and self-renewal exhaustion of hematopoietic stem cells in murine myelodysplastic syndrome. Proc Natl Acad Sci USA 107: 9783-9788, 2010.

7. Xia Q,Hu J and Meng YS: Abnormal expression of microRNA-124 in patients with leukemia or myelodysplastic syndrome and its significance. Zhongguo Shi Yan Xue Ye Xue Za Zhi 20: 358-361, 2012.

8. Castoro RJ, Dekmezian M, Saraf AJ, Watanabe Y, Chung W, Adhab SE, Jelinek J and Issa JP: MicroRNA 124 and its role in response to epigenetic therapy in patients with acute myelogenous leukemia and myelodysplastic syndrome. Blood 112: 598, 2008.

9. Lujambio A, Ropero S, BallestarE, Fraga MF, Cerrato C, Setién F, Casado S, Suarez-Gauthier A, Sanchez-Cespedes M, Git A, et al: Genetic unmasking of an epigenetically silenced microRNA in human cancer cells. Cancer Res 67: 1424-1429, 2007.

10. Tan P, Wei A, Mithraprabhu S, Cummings N, Liu HB, Perugini M, Reed K, Avery S, Patil S, Walker P, et al: Dual epigenetic targeting with panobinostat and azacitidine in acute myeloid leukemia and high-risk myelodysplastic syndrome. Blood Cancer J 4: e170, 2014.

11. Cheson BD, Greenberg PL, Bennett JM, Lowenberg B, Wijermans PW, Nimer SD, Pinto A, Beran M, de Witte TM, Stone RM, et al: Clinical application and proposal for modification of the International Working Group (IWG) response criteria in myelodysplasia. Blood 108: 419-425, 2006.

12. Livak KJ and Schmittgen TD: Analysis of relative gene expression data using real-time quantitative PCR and the 2 (-Delta Delta C(T)) Method. Methods 25: 402-408, 2001.

13. Liu HB, Mayes PA, Perlmutter P, McKendrick JJ and Dear AE: The anti-leukemic effect and molecular mechanisms of novel hydroxamate and benzamide histone deacetylase inhibitors with 5-aza-cytidine. Int J Oncol 38: 1421-1425, 2011.

14. Wong KY, So CC, Loong F, Chung LP, Lam WW, Liang R, Li GK, Jin DY and Chim CS: Epigenetic inactivation of the miR-124-1 in haematological malignancies. PLoS One 6: e19027, 2011.

15. Agirre X, Vilas-Zornoza A, Jiménez-Velasco A, Martin-Subero JI, Cordeu L, Gárate L, San José-Eneriz E, Abizanda G, Rodríguez-Otero P, Fortes $\mathrm{P}$, et al: Epigenetic silencing of the tumor suppressor microRNA Hsa-miR-124a regulates CDK6 expression and confers a poor prognosis in acute lymphoblastic leukemia. Cancer Res 69: 4443-4453, 2009.

16. Chen X, He D, Dong XD, Dong F, Wang J, Wang L, Tang J, Hu DN, Yan D and Tu L: MicroRNA-124a is epigenetically regulated and acts as a tumor suppressor by controlling multiple targets in uveal melanoma. Invest Ophthalmol Vis Sci 54: 2248-2256, 2013.

17. Chang CJ and Hung MC: The role of EZH2 in tumour progression. Br J Cancer 106: 243-247, 2012.

18. Sashida G, Harada H, Matsui H, Oshima M, Yui M, Harada Y, Tanaka S, Mochizuki-Kashio M, Wang C, Saraya A, et al: Ezh2 loss promotes development of myelodysplastic syndrome but attenuates its predisposition to leukaemic transformation. Nat Commun 5: 4177, 2014.

19. Herrera-Merchan A, Arranz L, Ligos JM, de Molina A, Dominguez $\mathrm{O}$ and Gonzalez S: Ectopic expression of the histone methyltransferase Ezh2 in haematopoietic stem cells causes myeloproliferative disease. Nat Commun 3: 623, 2012.

20. Tanaka S, Miyagi S, Sashida G, Chiba T, Yuan J, Mochizuki-Kashio M, Suzuki Y, Sugano S, Nakaseko C, Yokote K, et al: Ezh2 augments leukemogenicity by reinforcing differentiation blockage in acute myeloid leukemia. Blood 120: 1107-1117, 2012.

21. Agarwal P, Alzrigat M, Párraga AA, Enroth S, Singh U, Ungerstedt J, Österborg A, Brown PJ, Ma A, Jin J, et al: Genome-wide profiling of histone $\mathrm{H} 3$ lysine 27 and lysine 4 trimethylation in multiple myeloma reveals the importance of Polycomb gene targeting and highlights EZH2 as a potential therapeutic target. Oncotarget 7: 6809-6823, 2016.

22. Bose P, Simmons GL and Grant S: Cyclin-dependent kinase inhibitor therapy for hematologic malignancies. Expert Opin Investig Drugs 22: 723-738, 2013.

23. Fandy TE, Herman JG, Kerns P, Jiemjit A, Sugar EA, Choi SH, Yang AS, Aucott T, Dauses T, Odchimar-Reissig R, et al: Early epigenetic changes and DNA damage do not predict clinical response in an overlapping schedule of 5-azacytidine and entinostat in patients with myeloid malignancies. Blood 114: 2764-2773, 2009. 